WSRC-OS - - $-89-94-2$

DE92 011373

\title{
F/H AREA ETF EFFLUENT (H-016 OUTFALL) CERIODAPHNIA SURVIVAL/REPRODUCTION TEST, TEST DATE: SEPTEMBER 21, 1989 (U)
}

by

WSRC Contact: W. L. Specht

Westinghouse Savannah River Company

Savannah River Laboratory

Aiken, South Carolina 29808

This is a technical report being sent to OSTI and for distribution to the general public.

\section{DISCLAIMER}

\begin{abstract}
This report was prepared as an account of work sponsored by an agency of the United States Government. Neither the United States Government nor any agency thereof, nor any of their employees, makes any warranty, express or implied, or assumes any legal liability or responsibility for the accuracy, completeness, or usefulness of any information, apparatus, product, or process disclosed, or represents that its use would not infringe privately owned rights. Reference herein to any specific commercial product, process, or service by trade name, trademark, manufacturer, or otherwise does not necessarily constitute or imply its endorsement, recommendation, or favoring by the United States Government or any agency thereof. The views and opinions of authors expressed herein do not necessarily state or reflect those of the United States Government or any agency thereof.
\end{abstract}

The information contained in this article was developed during the course of work done under Contract No. DE-AC09-89SR18035 with the U.S. Department of Energy. By acceptance of this paper, the publisher and/or recipient acknowledges the U.S. Government's right to retain a nonexclusive, royalty-free license in and to any copyright covering this paper, along with the right to reproduce and to authorize others to reproduce all or part of the copyrighted paper. 
WS RC-OS-89.94.2

Derivative

Classifier

D. B. Moore-Shedrow, Section Manager

Authorized Derivative Classifer

F/H AREA ETF EFFLUENT (H-016 OUTFAL,L)

CERIODAPHNIA SURVIVAL/REPRODUCTION TEST,

TEST DATE: SEPTEMBER 21, 1989

(U)

WSRC Technical Representative: W. L. Specht

Approved

by

: Drome ftuer $\frac{}{\text { D. B. Moore-Shedrow, Section Manager }}$

Environmental Sciences Section

Savannah River Laboratory

Publication Date: August 1991

WESTINGHOUSE SAVANNAH RIVER COMPANY

SAVANNAH RIVER SIT:

AIKEN, SC 29808

Prepared for the U.S. Department of Energy under Contract No. DE-AC0.9-88SR18035

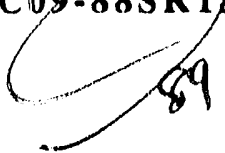

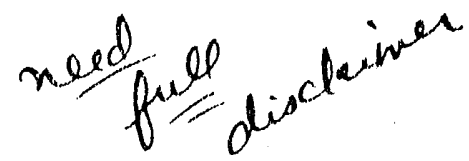




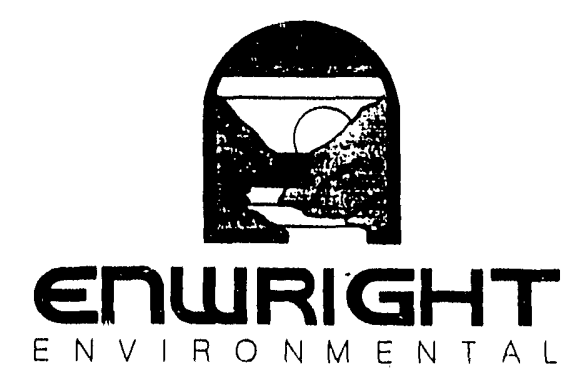

Ceriodaphnia SURVIVAL/REPRODUCTION TEST

Sample Identification: F/H Area ETF Ef $f$ luent

Client: Westinghouse Savannah River Company Location: Aiken, South Carolina

Test Date: $\quad$ September 21, 1989 
I NTERPRETATION OF RESULTS

This toxicity test was conducted to determine if the effluent causes death (acute toxicity) or reduction in the reproduction of the test organisms (chronic toxicity) during a seven day period. A series of dilutions of the effluent are set to determine how much the effluent must be diluted before toxic effects are no longer noted. Acute toxicity is checked by statistically analyzing whether significantly more organisms die in the effluent dilutions than in the control treatment, and, if significantly more die, how much the effluent must be diluted so as to kill only $50 \%$ of the test organisms (the LC50). Chronic toxicity is checked by statistically analyzing whether significantly fewer young are produced by test organisms exposed to the effluent dilutions. Results indicate the lowest effluent concentration which shows a toxic effect (the LOEC) and the highest effluent concentration which does not demonstrate an effect (NOEC). Results are summarized as follows:

\section{Acute Toxicity:}

Significant acute toxicity was present down to a concentration of 100.0\% ef fluent (by Fisher's Exact Test).

Probit Analysis

LC1 $=0.16 \%$ ef 1 luent
$L C 5=0.21 \%$ effluent
$L C 10=0.29 \%$ ef fluent
LC50 $=21.25 \%$ effluent

Fisher's Exact Test
$95 \%$ conf. int. lower upper

u 3.49

0 inf.

Chronic Toxicity:

Results based upon Bonferroni's " $t$ " Test are as follows:

( $95 \%$ confidence interval)

(NOEC) No Observed Effect. Concentration:

$30.0 \%$ effluent

(LOEC) Lowest Observed Effect Concentration: $100.0 \%$ effluent

NOEC $=30 \%$ ef fluent

LOEC $=100 \%$ effluent

Chronic Value:

$54.8 \%$ effluent 


\section{TES'T SYSTEM}

Client: Westinghouse Savannah River Company

Start Date: September 21, 1989

Test Type: 7 Day Chronic Toxicity - Reproduction Test

Test Location: Enwright Environmental Consulting Laboratories

Test Organism: Ceriodaphnia dubia Source: Enwright

Age: $\quad<24 \mathrm{hrs}$

Test Procedure: Short-Term Methods for Estimating the Chronic Toxicity of Effluents and Receiving Waters to Freshwater Organisms. 2nd Edition. EPA 600/4-89/001 Method 1002

Test vessels: $\quad 20 \mathrm{ml}$ plastic beakers

Amount Test Soln: $15 \mathrm{ml} /$ replicate

\# Replicates/Concentration: $\quad 10$ (test vessels randomized)

Teat nrganisms/Replicate: 1

\# of Test Concentrations Used (excluding control): 5

Test Solution Renewal: Daily

Food Source: 1 drop Selenastrum and 1 drop YCT daily / test vessel

Test Set By: Joel Stephens - Biologist

Test Results Checked By: Robert W. Kelley, Ph.D Biology Laboratory Manager 


\section{SAMPLING I NFORMATION}

Three effluent samples were collected by and transported to Enwright Environmental Consulting Labnratories by Enwright personnel. Samples were collected in $1 \mathrm{~L}$ plastic cubitainers and chilled during shipping.

Client: Westinghouse Savannah River Company Sample Identification: $\quad F / H$ Area ETF Effluent Start Date: $\quad$ September 21, 1989

\begin{tabular}{|c|c|c|c|}
\hline & $\begin{array}{l}\text { Sample } \\
\# 1\end{array}$ & $\begin{array}{l}\text { Sample } \\
\# 2\end{array}$ & $\begin{array}{l}\text { Sample } \\
\# 3\end{array}$ \\
\hline $\begin{array}{l}\text { Lab \# } \\
\text { Type }\end{array}$ & $\begin{array}{l}\text { BIO-259 } \\
\text { grab }\end{array}$ & $\begin{array}{l}\text { BIO-260 } \\
\text { grab }\end{array}$ & $\begin{array}{l}\text { BIO-261 } \\
\text { grab }\end{array}$ \\
\hline $\begin{array}{l}\text { Date sampled } \\
\text { Time sampled }\end{array}$ & Sept 20 & Sept 21 & Sept 25 \\
\hline $\begin{array}{l}\text { Days of use } \\
\text { Maximum hold time: }\end{array}$ & $\begin{array}{l}0 \\
24 \mathrm{hr}\end{array}$ & $\begin{array}{l}1,2,3 \\
72 \mathrm{hr}\end{array}$ & $\begin{array}{l}4,5,6 \\
48 \mathrm{hr}\end{array}$ \\
\hline Effluent Field Parameters & $\begin{array}{l}\text { Sample } \\
\# 1\end{array}$ & $\begin{array}{l}\text { Sample } \\
\# 2\end{array}$ & $\begin{array}{l}\text { Sample } \\
\# 3\end{array}$ \\
\hline $\begin{array}{l}\text { D. O. (ppm) } \\
\text { pH } \\
\text { Temp (C) } \\
\text { Res Cl (ppm) }\end{array}$ & \multicolumn{2}{|c|}{$\begin{array}{l}\text { No Data } \\
\text { Provided }\end{array}$} & $\begin{array}{r}9.0 \\
7.0 \\
21.0\end{array}$ \\
\hline
\end{tabular}




\section{RESULTS}

Client: Westinghouse Savannah River Company Sample Identification: F/H Area ETF Effluent Start Date: $\quad$ September 21, 1989

ACUTE TOXICITY (Effects on Survival)

\begin{tabular}{lcccccc}
\hline Conc. & $0.0 \%$ & $1.0 \%$ & $3.0 \%$ & $10.0 \%$ & $30.0 \%$ & $100.0 \%$ \\
\hline $\begin{array}{c}\text { Mortality } \\
\left(\begin{array}{l}7 \text { day }) \\
\text { Males }\end{array}\right.\end{array}$ & $0.0 \%$ & $20.0 \%$ & $10.0 \%$ & $30.0 \%$ & $30.0 \%$ & $100.0 \%$ \\
\hline
\end{tabular}

Significant acute toxicity was present down to a concentration of 100.0\% effluent (by Fisher's Exact Test). 
RESULTS

Client: Westinghouse Savannah River Company

Sample Identification:

F/H Area ETF Effluent

Start Date: September 21, 1989

CHRONIC TOXICITY - Daily Reproduction Totals

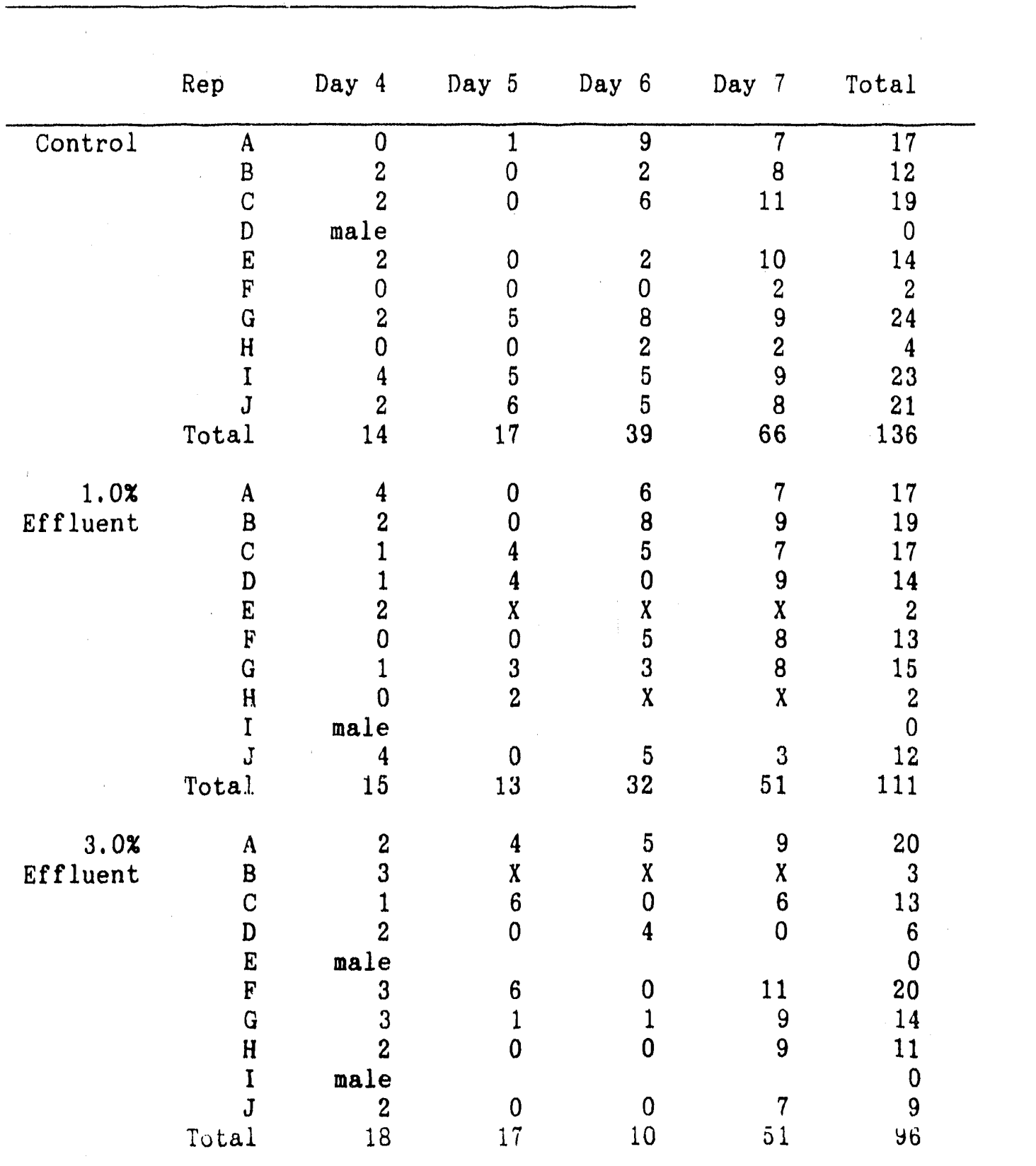

Note: Some control and effluent dilution organisms had fungal growth inhibiting reproduction. 
RESULTS

Client: Westinghouse Savannah River Company Sample Identification: $\quad F / H$ Area ETF Effluent Start Date: $\quad$ September 21, 1989

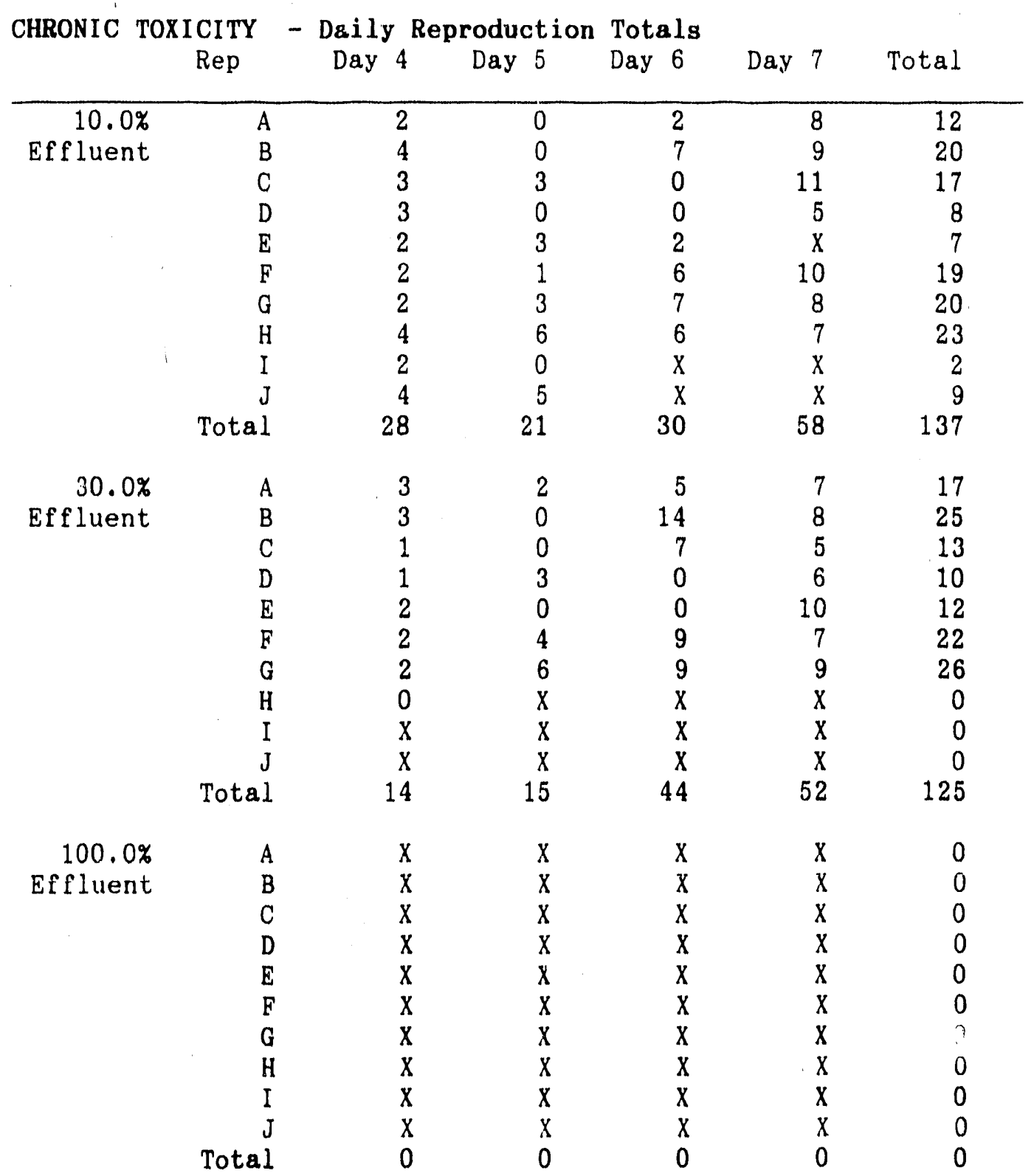

$X$ represents the mortality of the test organism. Note: The $100 \%$ effluent had $100 \%$ mortality on Day 1. Note: Some control and effluent dilution organisms hac fungal growth inhibiting reproduction. 


\section{RESULTS}

Client: Westinghouse Savannah River Company Sample Identification: F/H Area ETF Effluent Start Date: $\quad$ September 21, 1989

\section{CHRONIC TOXICITY - Statistical Analysis}

\begin{tabular}{|c|c|c|c|c|c|c|}
\hline Conc. & $0.0 \%$ & $1.0 \%$ & $3.0 \%$ & $10.0 \%$ & $30.0 \%$ & $100.0 \%$ \\
\hline $\begin{array}{c}\text { Average } \\
\text { Young/ } \\
\text { Female }\end{array}$ & 15.1 & 12.3 & 12.0 & 13.7 & 12.5 & 0.0 \\
\hline "t" Value & & 0.60 & 0.65 & 0.31 & 0.61 & \\
\hline $\begin{array}{l}\text { Reproduction } \\
\text { significantly less } \\
\text { than control? } \\
\text { ( } 95 \% \text { confidence) }\end{array}$ & & no & no & no & no & \\
\hline
\end{tabular}

$95 \%$ conf.

(NOEC) No Observed Effect Conc.: $30.0 \%$

(LOEC) Lowest Observed Effect Conc.: 100.0\%

Chronic Value:

$54.8 \%$

The data are normally distributed.

The data are homogenous in variance.

The number of replicates in each concentration is not equal.

The appropriate statistical procedure is Bonferroni's $t$ Test. 


\section{WATER CHEMISTRY ANALYSIS}

Client: Westinghouse Savannah River Company

Sample Identification: F/H Area ETF Effluent

Start Date: September 21, 1989

Dilution Water

ID: Upper Three Runs Creek (collected 9/21/89)

Preparation: Filtered ( 37 micron net)

Conductivity: 20 umhos

Hardness:

Alkalinity:

\section{Effluent}

ID: $\quad$ H/F Area ATF Area Effluent

Preparation: Filtered ( 37 micron net)

Hardness

Sample 1 Sample 2 Sample 3

Alkalinity

Conductivity

Res. Cl 


\section{WATER CHEMISTEZY ANALYSIS}

Client: Westinghouse Savannah River Company-

Sample Identification: F/H Area ETF Effluent

Start Date: $\quad$ September 21, 1989

Dilution Water

ID: $\quad$ Upper Three Runs Creek

Preparation: Filtered ( 37 micron net)

Conductivity: 20 umhos

Hardness: $\quad 6$

Alkalinit 4

Effluent

\begin{tabular}{|c|c|c|c|}
\hline $\begin{array}{l}\text { ID: H/F Area } \\
\text { Preparation: }\end{array}$ & $\begin{array}{l}\text { ATF Area } \\
\text { Filtered }\end{array}$ & $\begin{array}{l}\text { Effluent } \\
\text { (37 micro }\end{array}$ & n net) \\
\hline & Sample 1 & Sample 2 & Sam \\
\hline$i t$ & $\begin{array}{r}41 \\
2\end{array}$ & $\begin{array}{r}00 \\
2\end{array}$ & $\begin{array}{r}42 \\
2\end{array}$ \\
\hline tivity & 350 & 300 & 200 \\
\hline $\mathrm{Cl}$ & $<0.05$ & $<0.05$ & $<0.05$ \\
\hline
\end{tabular}


Client: Westinghouse Savannah River Company

Sample Identification: F/H Area ETF Effluent

Start Date: $\quad$ September 21, 1989

Test Solutions

CONTROL

D.O. D.O. Temp pH

(ppm) (\% sat.) (C)

Initial

Day 1 (old)

Day 1 (new)

Day 2 (old)

Day 2 (new)

Day 3 (old)

Day 3 (new)

Day 4 (old)

Day 4 (new)

Day 5 (old)

Day 5 (new)

Day 6 (old)

Day 6 (new)

Final

$1.0 \%$ Effluent

\begin{tabular}{rrrl}
8.3 & $101 \%$ & 24.2 & 7.3 \\
8.2 & $100 \%$ & & \\
8.4 & $102 \%$ & 24.0 & 7.4 \\
7.6 & $93 \%$ & & \\
7.4 & $90 \%$ & 24.0 & 7.7 \\
7.8 & $95 \%$ & & \\
7.4 & $94 \%$ & 25.6 & 6.7 \\
7.5 & $94 \%$ & & \\
7.6 & $95 \%$ & 25.1 & 7.5 \\
7.5 & $94 \%$ & & \\
7.6 & $95 \%$ & 25.1 & 7.5 \\
6.8 & $85 \%$ & & \\
7.5 & $95 \%$ & 25.7 & 7.7 \\
7.5 & $94 \%$ & 25.0 & 7.5 \\
\hline
\end{tabular}

$\begin{array}{ccc}\text { D.0. } & \text { D.0. } & \text { Temp } \\ (\mathrm{ppm}) & (\% \text { sat. }) & \text { (C) }\end{array} \quad$ pH

Initial

Day 1 (old)

Day 1 (new)

Day 2 (old)

Day 2 (new)

Day 3 (old)

Day 3 (new)

Day 4 (old)

Day 4 (new)

Day 5 (old)

Day 5 (new)

Day 6 (old)

Day 6 (new)

Final 
WATER CHEMISTRY ANALYSIS

Client: Westinghouse Savannah River Company Sample Identification: F/H Area ETF Effluent Start Date: $\quad$ September 21, 1989

\section{$3.0 \%$ Effluent}

Initial

Day 1 (old)

Day 1 (new)

Day 2 (old)

Day 2 (new)

Day 3 (old)

Day 3 (new)

Day 4 (old)

Day 4 (new)

Day 5 (old)

Day 5 (new)

Day 6 (old)

Day 6 (new)

Final

\section{$10.0 \%$ Effluent}

Initial

Day 1 (old)

Day 1 (new)

Day 2 (old)

Day 2 (new)

Day 3 (old)

Day 3 (new)

Day 4 (old)

Day 4 (new)

Day 5 (old)

Day 5 (new)

Day 6 (old)

Day 6 (new)

Final

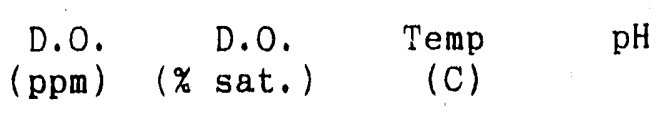

\begin{tabular}{rrrl}
8.3 & $101 \%$ & 24.2 & 7.3 \\
7.8 & $95 \%$ & & \\
8.6 & $105 \%$ & 24.0 & 7.3 \\
7.6 & $93 \%$ & & \\
7.7 & $94 \%$ & 24.0 & 7.6 \\
7.7 & $94 \%$ & & \\
7.8 & $99 \%$ & 25.6 & 6.9 \\
7.4 & $93 \%$ & & \\
7.6 & $95 \%$ & 25.1 & 7.4 \\
7.4 & $93 \%$ & & \\
7.6 & $95 \%$ & 25.1 & 7.4 \\
6.8 & $85 \%$ & & \\
7.5 & $95 \%$ & 25.7 & 7.1 \\
7.3 & $91 \%$ & 25.0 & 7.5 \\
\hline
\end{tabular}

D.0. D.0. Temp pH (ppm) (\% sat.) (C)

$\begin{array}{llll}8.3 & 101 \% & 24.2 & 7.3\end{array}$

$8.0 \quad 98 \%$

$\begin{array}{llll}8.6 & 105 \% & 24.0 & 7.3\end{array}$

$7.6 \quad 93 \%$

$\begin{array}{llll}7.2 & 88 \% & 24.0 & 8.1\end{array}$

$\begin{array}{lll}7.5 & 91 \% & \\ 7.8 & 99 \% & 25.6\end{array}$

$\begin{array}{llll}7.8 & 99 \% & 25.6 & 7.5\end{array}$

$\begin{array}{llll}7.4 & 93 \% & & \\ 7.6 & 95 \% & 25.1 & 7.4\end{array}$

$7.4 \quad 93 \%$

$\begin{array}{llll}7.6 & 95 \% & 25.1 & 7.4\end{array}$

$7.0 \quad 88 \%$

$\begin{array}{llll}7.5 & 95 \% & 25.7 & 7.2\end{array}$

$\begin{array}{llll}7.3 & 91 \% & 25.0 & 7.5\end{array}$ 
WATER CHEMISTRY ANALYSIS

Client: Westinghouse Savannah River Company

Sample Identification:

F/H Area ETF Effluent

Start Date: $\quad$ September 21, 1989

$30.0 \%$ Effluent

D.O. D.0. Temp $\quad \mathrm{pH}$

$(\mathrm{ppm})(\%$ sat. $) \quad$ (C)

Initial

Day 1 (old)

Day 1 (new)

Day 2 (old)

Day 2 (new)

Day 3 (old)

Day 3 (new)

Day 4 (old)

Day 4 (new)

Day 5 (old)

Day 5 (new)

Day 6 (old)

nay 6 (new)

Final

\begin{tabular}{rrrl}
8.3 & $101 \%$ & 24.2 & 7.4 \\
8.0 & $98 \%$ & & \\
8.4 & $102 \%$ & 24.0 & 7.4 \\
7.6 & $93 \%$ & & \\
7.4 & $90 \%$ & 24.0 & 7.9 \\
7.6 & $93 \%$ & & \\
8.0 & $101 \%$ & 25.6 & 7.6 \\
7.5 & $94 \%$ & & \\
7.0 & $88 \%$ & 25.1 & 7.4 \\
7.5 & $94 \%$ & & \\
7.0 & $88 \%$ & 25.1 & 7.4 \\
7.0 & $88 \%$ & & \\
7.5 & $95 \%$ & 25.7 & 7.3 \\
7.3 & $91 \%$ & 25.0 & 7.6 \\
\hline
\end{tabular}

$100.0 \%$ Effluent

$\begin{array}{lll}\text { D.0. } & \text { D.O. } & \text { Temp }\end{array} \quad$ pH

Initial

Day 1 (old)

Day 1 (new)

Day 2 (old)

Day 2 (new)

Day 3 (old)

Day 3 (new)

Day 4 (old)

Day 4 (new)

Day 5 (old)

Day 5 (new)

Day 6 (old)

Day 6 (new)

Final

$\begin{array}{llll}8.3 & 101 \% & 24.2 & 8.0 \\ 8.4 & 102 \% & 24.0 & 7.6\end{array}$




\section{QUALITY CONTROL}

Client: Westinghouse Savannah River Company

Sample Identification: F/H Area ETF Effluent

Start Date: September 21, 1989

\section{Culture Health:}

Sensitivity: Culture organisms were exposed to $\mathrm{NaCl}$ as a reference toxicant.

The 48 Hour LC50 for this test was $1.78 \mathrm{~g} / \mathrm{L}$

The mean $L C 50$ value at this laboratory for $\mathrm{NaCl}$ as a toxicant is $2.04 \mathrm{~g} / \mathrm{L}-+0.25$. The current LC50 of the culture organisins is 1.04 standard deviation units below the mean. This suggests the test organisms were slightly below the normal range.

Reproduction: The control test organisms produced a mean of

15.1 young over the 7 day test period. The mean number of young produced at this laboratory for Upper Three Runs Creek control tests is $17.0(+-3.5)$ young/female. Reproduction levels for this test were

0.54 standard deviation units below normal.

Test Precision: Mean Significant $D$ ifference (MSD):

The MSD for this test was 8.601 young. This is the least difference in the number of young produced by the control organisms vs. the number of young produced by any effluent treatment organisms which can be detected as statistically significant. 

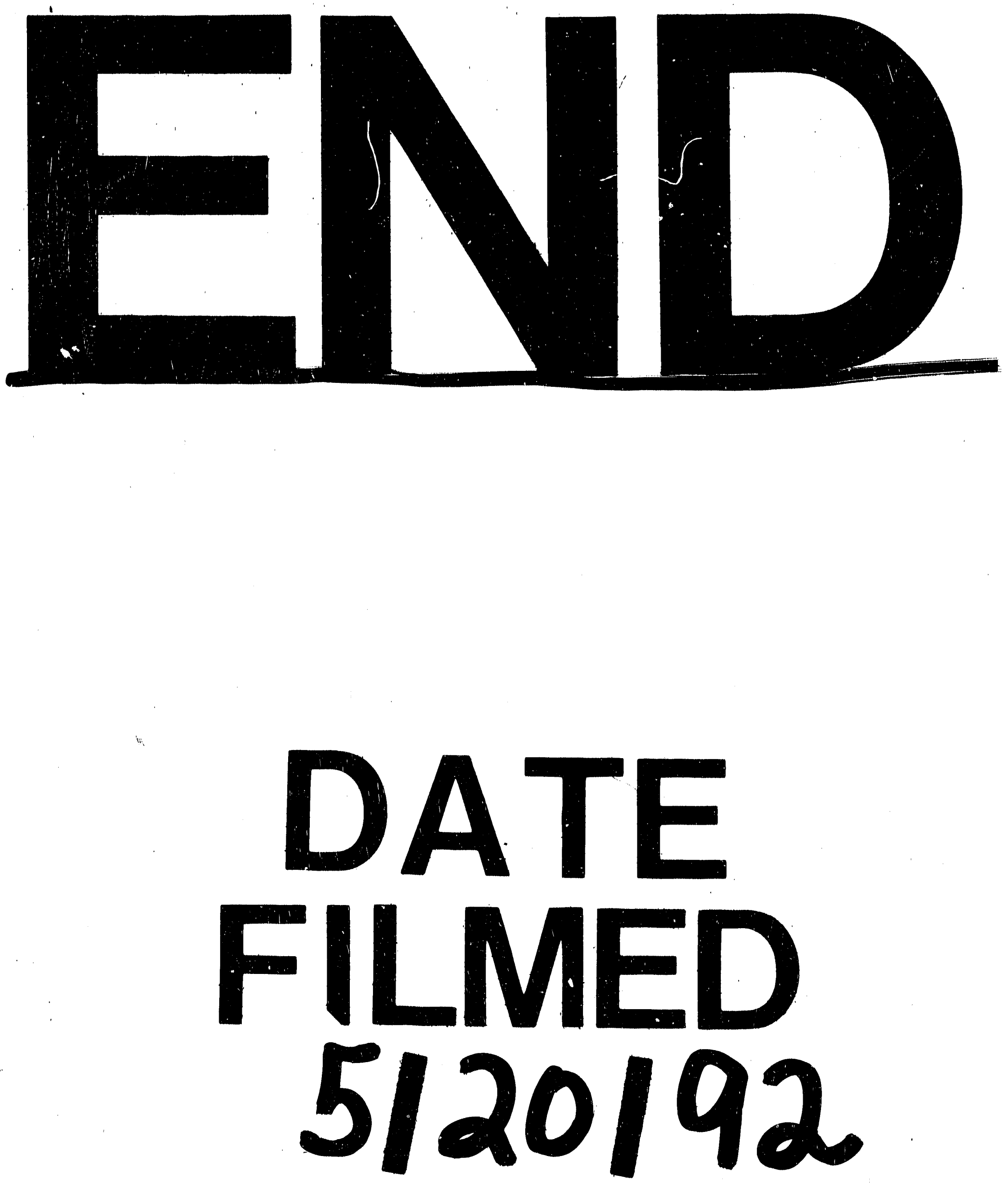
1 